\title{
EXPERIMENTS IN HUMAN VOLUNTEERS WITH TRACHOMA AND INCLUSION CONJUNCTIVITIS AGENT*†
}

BY

\author{
YUKIHIKO MITSUI, KATSUMOTO KONISHI, \\ AKIRA NISHIMURA, MASAHIRO KAJIMA, \\ OSAMU TAMURA, AND KUMIKO ENDO
}

From the Department of Ophthalmology, School of Medicine, Tokushima University, Japan

SINCE T'ang, Chang, Huang, and Wang (1957) reported success in trachoma virus cultivation in yolk sac of embryonate eggs, the cultivation of this virus and that of inclusion conjunctivitis has been re-examined and confirmed by a number of authors (Collier and Sowa, 1958; Collier, Duke-Elder, and Jones, 1958; Snyder, Page, Murray, Daggy, Bell, Nichols, Haddad, Hanna, and McComb, 1959; Hanna, Thygeson, Jawetz, and Dawson, 1959; Bernkopf, Nishmi, Maythar, and Feitelberg, 1959; Jones, Collier, and Smith, 1959; Grayston, Wang, Woolridge, Yang, and Johnston, 1960; Perret and Mann, 1960; Tarizzo, 1961). Volunteer experiments with the isolated agent have also been reported (T'ang and others, 1957; Collier and others, 1958, 1960; Bernkopf and others, 1959; Mann, Greer, Perret, and McLean, 1960; Jones and Collier, 1962; Thygeson, 1962), but these are very limited.

The present authors have undertaken experiments in a series of human volunteers with cultivated virus of the trachoma inclusion conjunctivitis (TRIC) group, and it is the purpose of this communication to discuss the clinical data of these experiments. The serology of the volunteers was examined by Dr. M. L. Tarizzo, Laboratoire de Récherches Virologiques, Centre Ophtalmologique, Tunis, and the results are to be reported in a separate paper (Tarizzo and Mitsui, in press).

\section{Isolation of Virus}

Four strains of TRIC virus were used for the volunteer experiments: three Japanese strains and one American strain. The American strain, Bourstrain, was that of trachoma originally isolated by Hanna and others (1959) in California. The three Japanese strains included two trachoma strains and one inclusion conjunctivitis strain isolated by the present authors. The isolation of these strains will briefly be discussed first.

\section{KONDO-STRAIN}

The donor patient was an 11-year-old boy, who had suffered from trachoma for several years. The clinical finding was typical chronic trachoma with superficial

\footnotetext{
* Received for publication December 12, 1961.

† Rhis investigation was supported by a Fight for Sight Grant-in-Aid of the National Council to Combat Blindness, Inc., New York.
} 
cicatrization, classified as $\operatorname{TrIII}+\mathrm{C}+++\mathrm{F}>\mathrm{PV} \pm$ according to the WHO classification. Typical inclusions were demonstrated in great number in the scrapings.

A conjunctival scraping was taken from the conjunctival fornix on May 30, 1960. It was mixed with $0.5 \mathrm{ml}$. sterile skim milk containing streptomycin in a concentration of $10 \mathrm{mg} . / \mathrm{ml}$. The mixture was kept at $+4^{\circ} \mathrm{C}$. for 4 hours, and was then inoculated into the yolk sac of four eggs incubated for 6 days. As shown in Table I, the embryos did not die for 7 days. On the 7th day they were opened and were passed into six eggs. The embryos showed a constant death after the passage. The elementary bodies in the yolk sac smear were negative in the original passage, but they turned positive after the passage.

TABLE I

ISOLATION OF TRIC-VIRUS FROM JAPANESE CASES

\begin{tabular}{c|c|c|c}
\hline \multirow{2}{*}{$\begin{array}{c}\text { Passage } \\
\text { No. }\end{array}$} & \multicolumn{3}{|c}{$\begin{array}{c}\text { No. of Eggs Died/Inoculated } \\
\text { (No. of Eggs showing Positive Elementary Bodies) }\end{array}$} \\
\cline { 2 - 4 } & KONDO-strain & MITA-strain & KAMI-strain \\
\hline 0 & $0 / 4(0)$ & $5 / 5(4)$ & $3 / 3(2)$ \\
1 & $6 / 6(6)$ & $6 / 6(5)$ & $5 / 5(5)$ \\
2 & $5 / 5(3)$ & $5 / 5(4)$ & $4 / 4(4)$ \\
3 & $5 / 5(5)$ & $3 / 3(3)$ & $8 / 8(7)$ \\
\hline
\end{tabular}

\section{Mita-Strain}

The donor patient was a 14-year-old girl, who had suffered from trachoma for 2 years. The clinical finding was typical chronic trachoma, classified as $\mathrm{TRII}+++\mathrm{F}>\mathrm{PV} \pm$. A few typical inclusions were demonstrated in the scrapings.

A conjunctival scraping was taken on June 16, 1960, and inoculated into five eggs as in the previous case. The embryos died in 7 to 9 days after inoculation, and elementary bodies were demonstrated in yolk sac smears from four of the five embryos (Table I). In subsequent passages the death of the embryos was constant, with positive elementary bodies.

\section{KAMI-STRAIN}

The donor patient was an 8-day-old baby suffering from inclusion blennorrhoea, first seen on the 5th day after delivery. The clinical picture was typical of neonatal inclusion blennorrhoea with a great number of inclusions.

A scraping was taken on December 24, 1960, and inoculated into three eggs as before. The embryos died in 7 to 9 days, and elementary bodies were shown in two of the three eggs. After a passage the embryos showed a constant death with positive elementary bodies (Table I).

\section{FAILURES}

Two other attempts at isolation from inclusion positive cases were discarded owing to bacterial contamination, and nine attempts from inclusion-negative cases gave no positive isolation of the virus. 


\section{Experiments in Volunteers}

Subjects.-Six volunteers hopelessly blind from intra-ocular disturbances but with normal conjunctivae and corneae were hospitalized and isolated at least for 2 weeks before the experiments. In four of the six volunteers, one strain of TRIC virus was inoculated into one eye. In two cases, one strain was inoculated into one eye and a different strain into the second eye simultaneously.

Technique of Inoculation.-10 per cent. emulsion of infected yolk sac was prepared with sterile skim milk. The emulsion was centrifuged at 4,000 r.p.m. for 15 minutes. One drop of the supernatant was instilled into the cul-de-sac. No mechanical injuries such as scarification were applied to the conjunctiva at the time of inoculation. In the unilateral experiments a bandage was placed on the eye until the onset of the disease, and in the bilateral cases until the beginning of treatment.

Identification of Re-isolation of the Virus.-A re-isolation of the virus was tried in chick-embryo from some of the volunteers. Positive isolation was recognized by constant death of the embryo with positive inclusions in a section of the yolk sac (see Fig. 5, below) according to the procedure reported elsewhere (Mitsui and others, 1962).

\section{Results}

The results of the volunteer experiments are summarized in Table II.

TABLE II

RESULTS OF SIX VOLUNTEER EXPERIMENTS

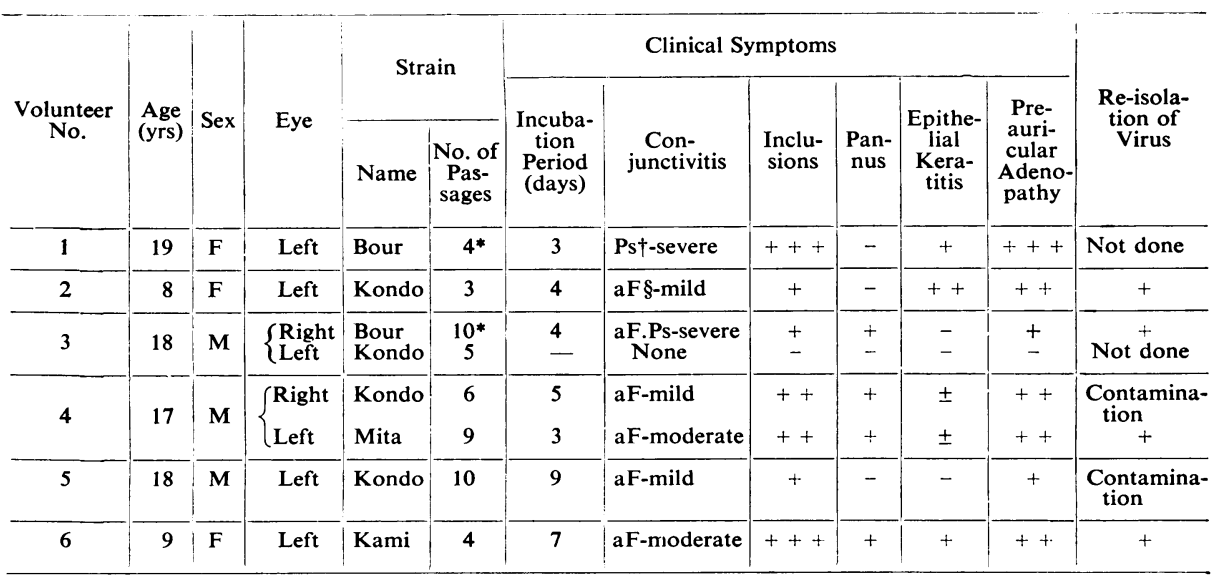

* No. of passage of Bour-strain is that after arrival in the authors' laboratory

$\uparrow$ Ps: Pseudomembranous conjunctivitis

$\S$ aF: Acute follicular conjunctivitis 
Case 1, a 19-year-old female (Fig. 1), had Bour-strain inoculated into the left eye on December 11, 1959, after four passages in our laboratory. After 3 days' incubation, an acute conjunctivitis resulted in the inoculated eye. The inflammation increased rapidly and, in a few days, acute follicular pseudomembranous conjunctivitis developed, and the lids were swollen. A tender pre-auricular adenopathy appeared, and there was also swelling of the cervical lymph nodes. Numerous inclusions were demonstrated in every examination after the onset of the disease (Fig. 2), but no bacteria were found. A slight and scattered epithelial keratitis appeared on the 3rd day of the disease but no pannus appeared up to the end of the observation period.

On the 8th day of the disease, a severe pseudomembranous conjunctivitis was seen, and the volunteer complained of pain in the swollen cervical lymph nodes. Treatment was started on the 9th day, 1 per cent. tetracycline ointment being applied topically three times a day with oral sulphadiazine, $1.5 \mathrm{~g}$. daily divided into three doses. The inflammation subsided rapidly, and after 4 weeks the conjunctiva appeared to be normal.

The inflammation did not spread to the second eye.

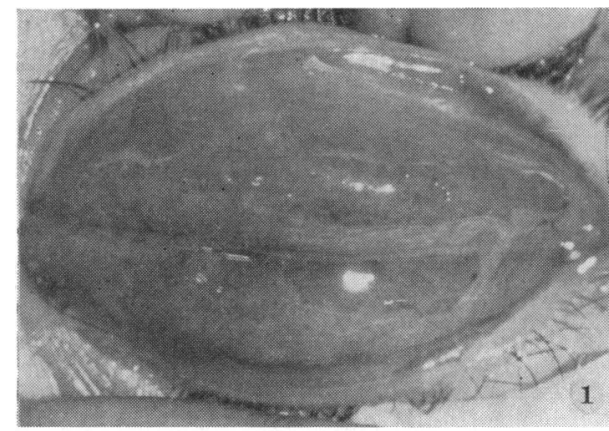

Fig. 1.-Case 1, left eye (inoculated with Bourstrain). 8th day after onset of disease.

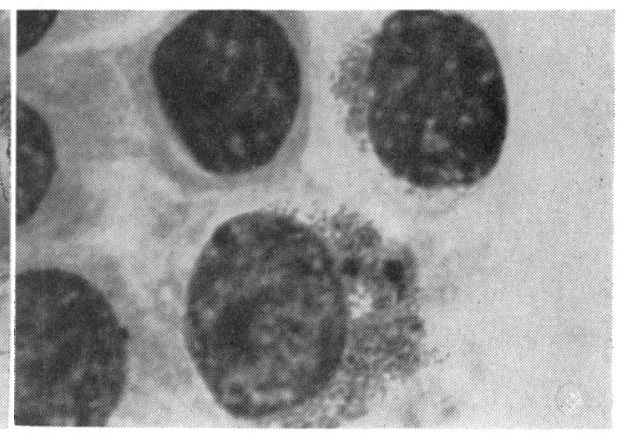

Fig. 2.-Inclusions in scraping from Case 1 (inoculated with Bour-strain).

Case 2, an 8-year-old girl (Fig. 3), had Kondo-strain inoculated into the left eye on June 22,1960 , after three passages through the yolk sac. After 4 days' incubation, an acute conjunctivitis resulted in the inoculated eye; pre-auricular adenopathy appeared on the second day of the disease, and inclusions were positive in a scraping on the same day (Fig. 4, opposite), but no bacteria were found. The inflammation increased slowly and after one week a mild but typical acute follicular conjunctivitis had developed. The virus was successfully re-isolated from material taken on the 10th day (Fig. 5, opposite).

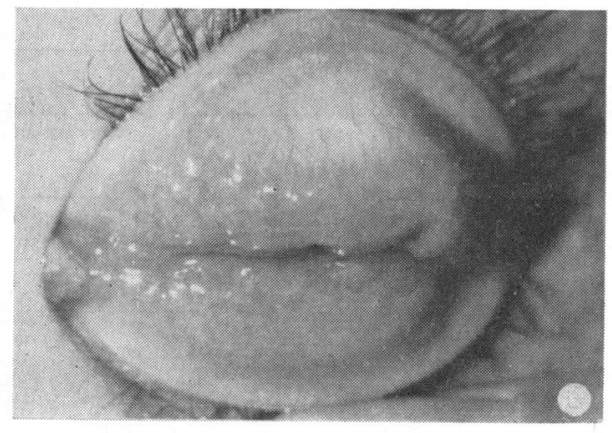

Fig. 3.-Case 2, left eye (inoculated with Kondo-strain) 12th day after onset of disease. 


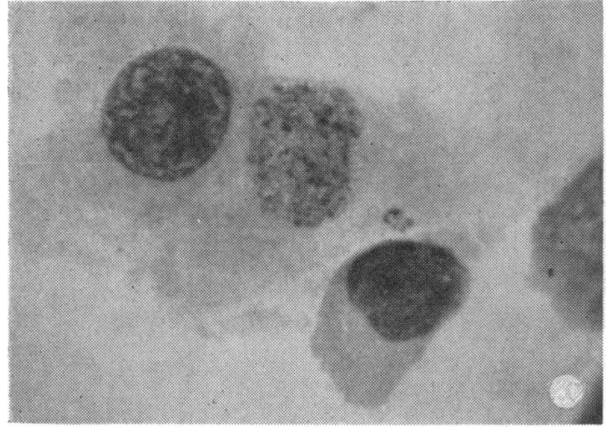

FIG. 4.-Inclusions in scraping from Case 2 (inoculated with Kondo-strain).

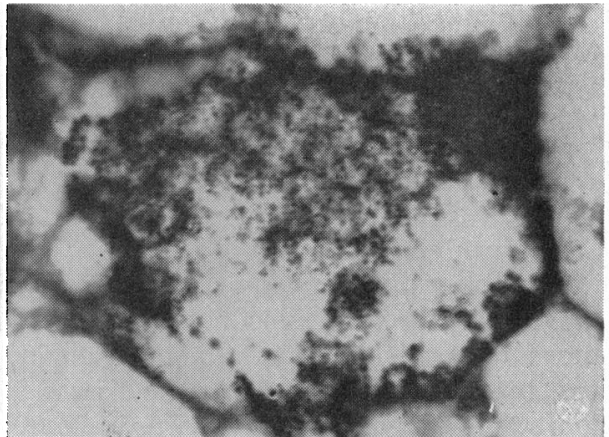

FIG. 5.-Inclusion in yolk sac section. Reisolated virus from Case 2 (inoculated with Kondo-strain).

In the 2nd week an epithelial keratitis appeared at the lower part of the cornea. There was a dilatation of the limbal capillaries at the upper limbus but the vessels did not extend into the cornea up to the end of the 4 weeks' observation period.

The acute inflammation began to subside during the 4th week. The same treatment was given as in the previous case from the 5th week; after one month the conjunctiva was almost normal except for a slight hyperaemia, and 3 months later it was perfectly normal.

The inflammation was confined to the one eye throughout.

Case 3, an 18-year-old male (Fig. 6), had Bour-strain inoculated into his right eye after ten passages in our laboratory, and Kondo-strain in the left eye after five passages, both on September 5, 1960.

After 4 days' incubation, an acute conjunctivitis resulted in the right eye (Bour-strain). The inflammation increased rapidly and, on the 3rd day of the disease, the clinical picture was that of an acute follicular conjunctivitis. Pre-auricular adenopathy appeared on the 4th day of the disease, and microscopic but definite pannus and pseudomembrane on the 7 th day.

FIG. 6.-Case 3, right eye (inoculated with Bour-strain). 7 th day after onset of disease.

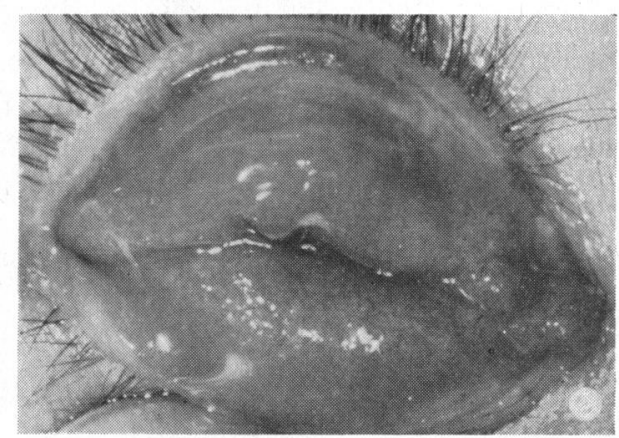

A few inclusions were demonstrated on the 8th day, but no bacteria were found; the virus was successfully re-isolated from a conjunctival scraping. An acute and severe follicular pseudomembranous conjunctivitis was seen 2 weeks after the onset of the disease. The pseudomembrane disappeared by the end of the 3rd week, and during the 4 th week the acute signs began gradually to subside. No epithelial keratitis was seen. 
From the beginning of the 5th week, treatment was given as in the previous cases. After 4 weeks' treatment, the clinical appearance was that of folliculosis with a trace of hyperaemia, and 3 months later a few transparent follicles remained in the lower lid without inflammation.

In the left eye, which was inoculated with Kondo-strain, there was no evidence of "take" up to the end of the observation period.

Case 4, a 17-year-old male (Figs 7 and 8), had Kondo-strain inoculated into the right eye after six passages, and Mita-strain into the left eye after nine passages, both on November 5,1960 . After 3 days' incubation, the left eye (Mita-strain) showed signs of "take", and after 5 days a typical acute follicular conjunctivitis was seen. Pre-auricular adenopathy was apparent on the 2nd day of the disease, and inclusions were positive on the 7th day, but no bacteria were found. The virus was successfully re-isolated from material taken on the 7th day. A microscopic pannus appeared at the upper limbus in 10 days. The inflammation reached its climax in 2 weeks with a moderately severe acute follicular conjunctivitis. Transient epithelial keratitis was noted at the upper cornea by the end of the 2nd week.

In the right eye (Kondo-strain) a sign of "take" was apparent after 5 days' incubation. The course of the disease was similar to that of the left eye, but the inflammation was milder and the progress slower. Microscopic pannus was apparent, but the epithelial keratitis was very slight and transitory.

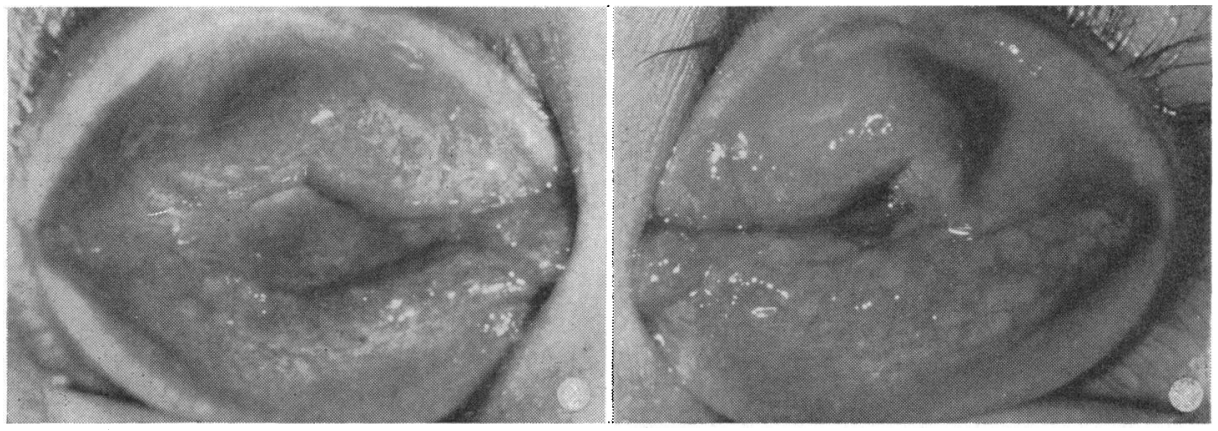

Fig. 7.-Case 4, right eye (inoculated with Kondo-strain). 20th day after onset of

FIG. 8.-Case 4, left eye (inoculated with disease.

Mita-strain). 22nd day after onset of disease.

The inflammation reached its climax in both eyes 4 weeks after the onset of the disease and the acute signs began gradually to subside.

By the middle of the 5th week, the patient was given an intra-muscular injection of Lederkyn suspension (equivalent to $2.5 \mathrm{~g}$. Lederkyn), and this was repeated three times in intervals of 8 and 14 days. The response was prompt, and 1 month after the first injection, there only remained a small number of follicles without inflammation; 3 months later there were still a few transparent follicles but no signs of trachoma.

Case 5, an 18-year-old male (Fig. 9, opposite), had Kondo-strain inoculated into the left eye after ten passages, on January 17,1961. After 9 days' incubation, an acute conjunctivitis appeared and one week later there was mild but acute follicular conjunctivitis. A slight pre-auricular adenopathy appeared after 5 days. Inclusions were positive on the 6th day, but no bacteria were found. Pannus and epithelial keratitis were negative up to the end of the observation period.

After reaching a climax by the end of the 2 nd week, the inflammation gradually reached a chronic stage. 
Fig. 9.-Case 5, left eye (inoculated with Kondo-strain), 13th day after onset of disease.

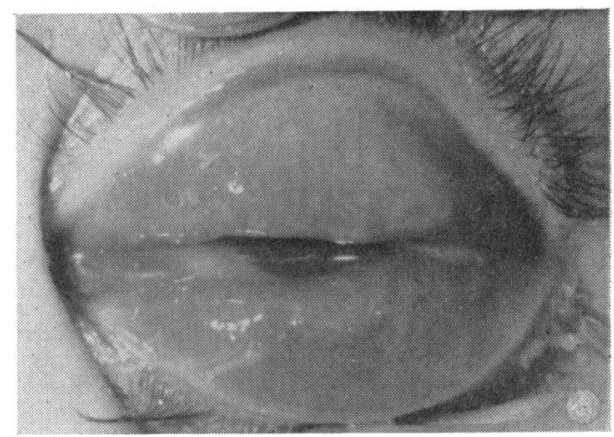

From the beginning of the 5th week, Lederkyn was given intramuscularly as in the previous case, and the injection was repeated three times at intervals of 7 and 8 days. 3 weeks after the first injection the conjunctiva was almost normal except for a few follicles, and these disappeared during the following month.

The right eye remained unaffected throughout.

Case 6, a 9-year-old female (Figs 10 and 11), had Kami-strain inoculated into her left eye after four passages, on January 31, 1961. After 7 days' incubation, an acute conjunctivitis started and increased rapidly until in 5 days typical acute follicular conjunctivitis was seen. A tender pre-auricular adenopathy appeared on the 3rd day. Inclusions were seen on the 6th day, but no bacteria were found. The virus was successfully re-isolated from scrapings taken on the 6th day. After 10 days, a microscopic pannus and epithelial keratitis began to appear at the upper limbus. After 2 weeks, there was a moderately severe acute follicular conjunctivitis, and the pannus extended $1 \mathrm{~mm}$. into the cornea beyond the scleral band.

The symptoms remained unchanged during the 3 rd week, but began gradually to subside during the 4th week. Intramuscular Lederkyn was given in the 5th week, and after a 15-day interval. 4 weeks after treatment was completed a trace of follicles and papillae remained, but 2 months later the conjunctiva appeared completely normal.

The right eye remained normal up to the end of the observation period.

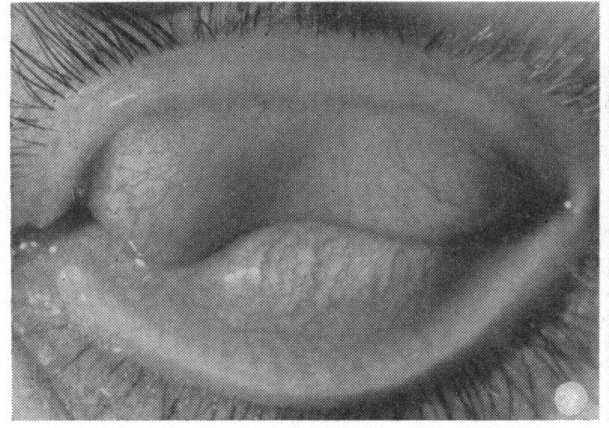

Fig. 10.-Case 6, left eye (inoculated with Kami-strain), before inoculation.

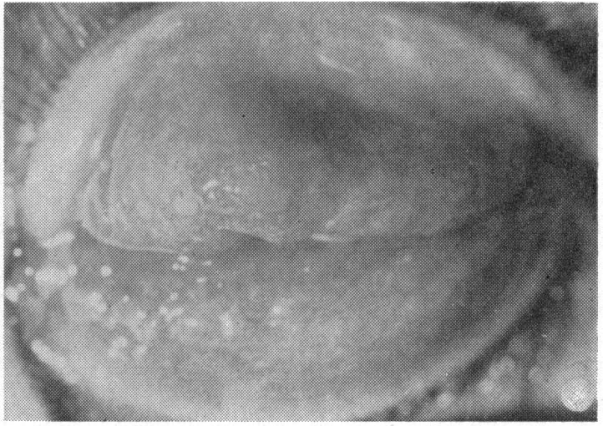

Fig. 11.-Case 6, left eye on 18th day after onset of disease.

\section{Discussion}

Eight eyes of six volunteers were inoculated with egg-grown TRIC virus. One drop of 10 per cent. yolk sac emulsion was instilled into each eye. The 
inoculum contained approximately $10^{3}-10^{4} \mathrm{EID} 50 / \mathrm{ml}$. of the virus. No mechanical injury was done to the conjunctiva at the time of inoculation. Seven of the eight eyes showed a definite "take".

The clinical characteristics of this trachoma induced by inoculation with egg-grown virus were almost the same as those of experimental trachoma induced by inoculation with patient's material as well as those of acute trachoma due to natural infection (Mitsui, 1949). The onset of the disease was acute in all cases. The clinical picture of the acute stage was that of an acute follicular conjunctivitis. Severe cases developed pseudomembranous conjunctivitis. Pannus, epithelial keratitis, and pre-auricular adenopathy were seen, but did not invariably occur. The only indispensable symptom of trachoma at the onset was the presence of "positive inclusions" in scrapings.

The severity of the symptoms differed in different cases (Table III), according to the susceptibility of the volunteers. Each of the two strains, Bour and Kondo, was inoculated into two or more volunteers, but did not always provoke the same symptoms, so that the difference in severity does not necessarily indicate a "strain-difference". Nevertheless, our results suggest the possible presence of a "strain-difference". One volunteer (Case 4) was inoculated with two different strains simultaneously, one into each eye. There was a definite difference between the two eyes in the severity of symptoms.

TABLE III

STRAIN AND SEVERITY OF CLINICAL SYMPTOMS

\begin{tabular}{l|l|c|l}
\hline \multicolumn{1}{c|}{ Strain } & \multicolumn{1}{c|}{ Origin } & Eye Number & \multicolumn{1}{c}{ Severity } \\
\hline Bour & Trachoma (U.S.A.) & 1 & $\begin{array}{l}\text { Extremely severe } \\
\text { Severe }\end{array}$ \\
\hline Mita & Trachoma (Japan) & 1 & Moderate \\
\hline Kami & Inclusion Blennorrhoea (Japan) & 1 & Moderate \\
\hline Kondo & Trachoma (Japan) & 1 & Mild \\
& & 2 & Mild "take" \\
& & 3 & No “take \\
\hline
\end{tabular}

So far, the Bour-strain seems to be extremely virulent. In our extensive experience of volunteer experiments with TRIC virus (Mitsui, 1949), we had never seen such severe manifestations as were caused by this strain.

The Kondo-strain seems to be the least virulent. One inoculation failed, and this was not due to the insusceptibility of the volunteer, since the other eye reacted to the Bour-strain which was inoculated at the same time. Three other inoculations with Kondo-strain resulted in only a "mild" acute follicular conjunctivitis. 
The Mita- and Kami-strain seemed to be moderately virulent.

In considering virulence, the number of egg-passages should also be borne in mind. So far, however, as suggested by three successful inoculations with Kondo-strain after different numbers of egg-passages, as many as ten passages do not seem to affect the virulence of the virus.

One trachoma strain (Mita) and one inclusion conjunctivitis strain (Kami) showed much the same degree of virulence, with similar clinical symptoms of about the same severity. Pannus occurred with both these strains.

\section{Summary and Conclusions}

The isolation of two strains of trachoma virus and one strain of inclusion conjunctivitis virus from Japanese cases of trachoma and inclusion blenorrhoea is described. The results of human inoculations with these strains and with one American strain are discussed. A quantitative difference in the severity of clinical symptoms due to different strains is likely to be present, but no qualitative difference was demonstrated among the strains, even between trachoma and inclusion conjunctivitis.

\section{REFERENCES}

Bernkopf, H., Nishmi, M., Maythar, B., and Feitelberg, I. (1959). A.M.A. Arch. Ophthal., $62,33$.

Collier, L. H., Duke-Elder, S., and Jones, B. R. (1958). Brit. J. Ophthal., 42, 705. (1960). Brit. J. Ophthal., 44, 65. and Sowa, J. (1958). Lancet, $1,993$.

Grayston, J. T., WANG, S. P., Woolridge, R. L., YANG, Y. F., and Johnston, P. B. (1960). J. Amer. med. Ass., 172, 1577.

Hanna, L., Thygeson, P., Jawetz, E., and Dawson, C. (1959). Science, 130, 1339.

Jones, B. R., and Collier, L. H. (1962). Ann. N.Y. Acad. Sci., 98, 212.



ManN, I., Greer, C. H., Perret, D., and McLean, C. (1960). Brit. J. Ophthal., 44, 641.

MrTsui, Y. (1949). Amer. J. Ophthal., 32, 1189.

KaJima, M., Nishimura, A., and Konishi, K. (1962). Ann. N.Y. Acad. Sci., 98, 131.

Perret, D., and MANN, I. (1960). Brit. J. Ophthal., 44, 503.

SNYder, J. C., Page, R. C., Murray, E. S., DAGGY, R. H., Bell, S. D., Nichols, R. L., Haddad, N. A., Hanna, A. T., and McComb, D. (1959). Amer. J. Ophthal., 48, No. 3 Pt. II, p. 325.

T'ANG, G., Chang, H., HuAng, Y., and Wang, K. (1957). Chin. med. J., 75, 429.

TARIzZo, M. L. (1961). Bull. Wld Hlth Org., 24, 103.

and MrTsuI, Y. (in press). Ibid.

Thygeson, P. (1962). Ann. N.Y. Acad. Sci., 98, 201. 